\title{
Laser-induced thermal acoustics: simple accurate gas measurements
}

\author{
E. B. Cummings \\ Graduate Aeronautical Laboratories, California Institute of Technology, Pasadena, California 91125
}

Received February 17, 1994

\begin{abstract}
Laser-induced thermal acoustics (LITA), an optical four-wave mixing technique, has been used for sensitive measurement of the sound speed, thermal diffusivity, acoustic damping rate, and complex susceptibility of a gas. In LITA, laser-induced acoustic waves scatter laser light into a coherent, modulated signal beam. A simple expression accurately describes the signal. Atmospheric sound speeds accurate to $0.5 \%$ and transport properties accurate to $30 \%$ have been measured in a single shot without calibration. LITA spectra have been taken of weak spectral lines of $\mathrm{NO}_{2}$ in concentrations of less than 50 parts in $10^{9}$. Signal reflectivities up to $10^{-4}$ are estimated.
\end{abstract}

A four-wave mixing (FWM) technique that the author proposes to call laser-induced thermal acoustics (LITA) has been applied for single-shot point measurement of sound speed and thereby of temperature, if the gas composition is known), thermal diffusivity, and acoustic damping (and thereby of viscosity) of a gas. ${ }^{1}$ LITA signals arise from a sequence of optoacoustic and acousto-optic effects, enhanced by quenching. LITA's diverse capabilities, including single-shot gas property, velocity, and multiplex spectra measurement, portend a robust line of research and application. A similar technique has been used to measure sound speeds in crystals. ${ }^{2}$ Buntine et al. ${ }^{3}$ first observed LITA signals from gases in delayedpump FWM experiments, ${ }^{3}$ and several groups of scientists are studying thermal gratings (used in LITA) in connection with degenerate FWM.

With cw argon-ion laser scattering off acoustic waves excited by a 5-ns dye laser, the temperature of the laboratory air was measured by uncalibrated, single-shot LITA within $0.5 \%$, limited by uncertainty of the air composition and beam geometry. Transport properties were measured within $30 \%$ of published values, limited by a finite beam-size effect, which was ignored. The two optoacoustic effects of electrostrictiction and thermalization, proportional to the real and the imaginary parts of the gas susceptibility, respectively, produce different LITA signals, permitting sorting of each effect. In this way LITA was used to measure a spectrum of the complex gas susceptibility showing weak visible lines of the laboratory-air trace species $\mathrm{NO}_{2}$ (less than 50 parts in $10^{9}$ ). The absolute amplitude and time history of LITA signals are accurately represented by predictions of an analytical expression derived previously from the linearized equations of light scattering and hydrodynamics. ${ }^{1}$

LITA is a laser-induced dynamic grating technique that employs a combination of optoacoustic and acousto-optic effects. Light from a short-pulse driver laser (e.g., <10 ns) generates acoustic waves by electrostriction and rapid thermalization. Electrostriction, the tendency of polarizable molecules to move toward regions of high optical electric-field intensity, ${ }^{4}$ is proportional to the real part of the gas susceptibility. Thermalization is a multiple-step collisional process proportional to the imaginary part of the gas susceptibility: molecules of the gas absorb driver-laser energy; inelastic collisions convert part of the excited-state energy into molecular kinetic or rotational energy; finally, elastic molecular collisions equilibrate the velocities of the molecules, raising the temperature. Acoustic waves are produced efficiently when the drop in gas density accompanying thermalization sharply lags that of equilibration. The density-perturbation field of the acoustic waves generates a susceptibility-perturbation field that scatters light from a long-pulse or cw source laser, forming the LITA signal.

A forward-scattering FWM has been used for LITA. The driver laser is split into two beams, which cross at a small angle $(0.5-5 \mathrm{deg})$ in the gas, where they interfere with each other, forming an ellipsoidal electric-field intensity grating at their intersection. Here, optoacoustic effects generate acoustic structures with modulation depths from $0.01 \%$ to $5 \%$. Electrostiction forms two traveling acoustic wave packets (phonons) and a weak stationary isobaric density grating (thermon), each with the wavelength of the electric-field grating. Thermalization generates similar acoustic structures, but the thermon is dominant. The phonons counterpropagate in the direction parallel to the grating vector and decay by acoustic damping, whereas the thermon (stationary with respect to the fluid) decays by heat conduction. Light scattered from the source laser by the acoustic gratings emerges in a coherent signal beam when the source laser is incident at the Bragg angle of the grating. Light scattered by the phonons has Doppler shifts relative to light scattered by the thermon of plus and minus the Brillouin frequency, $\omega_{B}=c_{s} q$, $q \equiv 2 k_{d} \sin (\theta / 2)$, where $c_{\mathrm{s}}$ is the speed of sound, $q$ is the grating vector magnitude, $\theta$ is the driver-beam crossing angle, and $k_{d}$ is the driver-beam wave-vector magnitude. Beating between the light scattered by these structures modulates the detected signal, which 


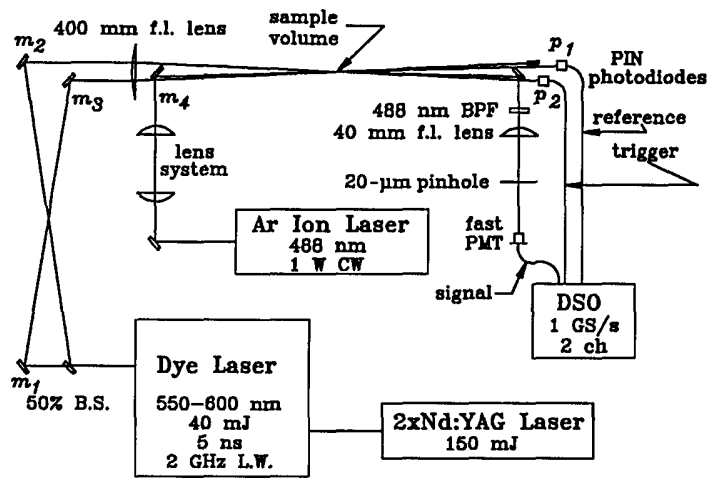

Fig. 1. LITA experimental setup: f.l., focal length; $\mathrm{BPF}$, bandpass filter.

bears a dc component; a component at the Brillouin frequency caused by phonon/thermon signal beating; and a component at twice the Brillouin frequency caused by beating of signals generated by the conjugate phonons.

Current experiments detect the relative Doppler shifts of light scattered from a narrow-band source laser. However, convection of the phonons and thermon causes a common Doppler shift in the signal beam that can be measured by optical heterodyne techniques, permitting velocimetry by analogy with laser-Doppler velocimetry. One may also use LITA for single-shot spectral measurements by spectrally resolving the LITA signal scattered from a broadband source laser. Experiments to study both of these LITA capabilities are under development.

An analytical expression previously derived for the amplitude and time history of the LITA signal allows the magnitude of LITA signals to be estimated $a$ priori and the actual LITA signal to be interpreted quantitatively, ${ }^{1}$ with very good experimental agreement. The analysis treats the optoacoustic effects and the hydrodynamic evolution of the acoustic structures, using the linearized hydrodynamic equations. ${ }^{5}$ The perturbation solution for the far field of light scattered off a dielectric disturbance accounts for the acousto-optical effect. ${ }^{5}$ A simple analytical result for the signal intensity, $I(t)$, is obtained for a short-duration Gaussian driver beam, infinite-extent, monochromatic source beams, and single-rate-dominated thermalization: ${ }^{1}$

$I(t) \propto\left[A \exp \left(-D_{T} q^{2} t\right)+B \cos \left(c_{s} q t+\Phi\right) \exp \left(-\Gamma q^{2} t\right)\right]^{2}$,

where $D_{T}$ is the thermal diffusivity, $\Gamma$ is the acoustic damping coefficient, and $A, B$, and $\Phi$ are simple algebraic functions of gas and laser-beam parameters. When the approximations listed above are not valid, spatial and temporal convolutions of laser-beam profiles and thermalization behavior augment the analysis. The ratio of signal-beam to source-beam intensity is of the order of $10^{-4}$ for room air with a thermal modulation depth of $1 \%$ in a 2-mm-long sample volume, without resonant enhancement at the source-laser wavelength. ${ }^{1}$

Figure 1 is a schematic diagram of the experimental apparatus used in these LITA experiments. The driver laser is a 2-GHz-linewidth (L.W.) dye laser, which emits $\sim 40 \mathrm{~mJ}$ of light tuned between 587 and $592 \mathrm{~nm}$ in 5-ns pulses. 3-mm-wide driver beams are formed from the dye laser emission by a $50 \%$ beam splitter (B.S.) and a mirror $\left(\mathrm{m}_{1}\right)$. The driver beams intersect at the focus of the $400-\mathrm{mm}$ lens, where they approximate finite plane waves $\sim 200 \mu \mathrm{m}$ in diameter. The beam-crossing angle is set by the distance between the parallel beams, which is adjusted by use of micrometer translation stages under mirrors $\mathrm{m}_{2}$ and $\mathrm{m}_{3}$. Three-dimensional BOXCARS geometries ${ }^{6}$ with crossing angles ranging from $1.0^{\circ}$ to $2.3^{\circ}$ were used in these experiments. The driver-beam pulse triggers the acquisition of the LITA signal by means of silicon P-I-N photodiode $p_{1}$ (Thor Labs Model DET2SI). The source laser is a 1-W cw argon-ion laser operating at $488 \mathrm{~nm}$ (Spectra-Physics Model 165). A two-lens system adjusts the source beam diameter, which ranged from $\sim 200 \mu \mathrm{m}$ to $2 \mathrm{~mm}$. Mirror $\mathrm{m}_{4}$ points the source beam to the sample volume. A silicon P-I-N photodiode, $\mathrm{p}_{2}$, monitors the intensity of the source beam.

The detection system consists of a fast photomultiplier tube (PMT) (Hammamatsu Model OPTO-8) with at least a 500-MHz signal bandwidth and optical filters to prevent signal contamination and saturation, including a $10-\mathrm{nm}$ interference filter at $488 \mathrm{~nm}$ and a $40-\mathrm{mm}$ lens $20-\mu \mathrm{m}$ pinhole spatial filter. The PMT dynode voltages are maintained during the signal pulse by Mylar and silver-mica capacitors with average lead lengths less than $2 \mathrm{~cm}$. The LITA signal path length to the detector is $\sim 2.5 \mathrm{~m}$. Data are recorded with a 1-GS/s, 8-bit, two-channel digital storage oscilloscope (DSO, HP Model 54510A). This records typically 500-2000 sample time histories of signals from the PMT and $p_{2}$.

In experiments using laboratory air, completely nonresonant electrostriction generated a LITA signal of roughly 30 photons $/ \mathrm{ns}$ (reflectivity $\sim 10^{-8}$ with the 1 -W source beam). An average of 256 shots yields the nonresonant data plotted as the dotted curve in Fig. 2. The frequency of the modulation is $\sim 26 \mathrm{MHz}$, twice the Brillouin frequency, yielding a sound speed accurate within the uncertainty of the knowledge of the laboratory sound speed $(\sim 0.5 \%)$. The resonantly

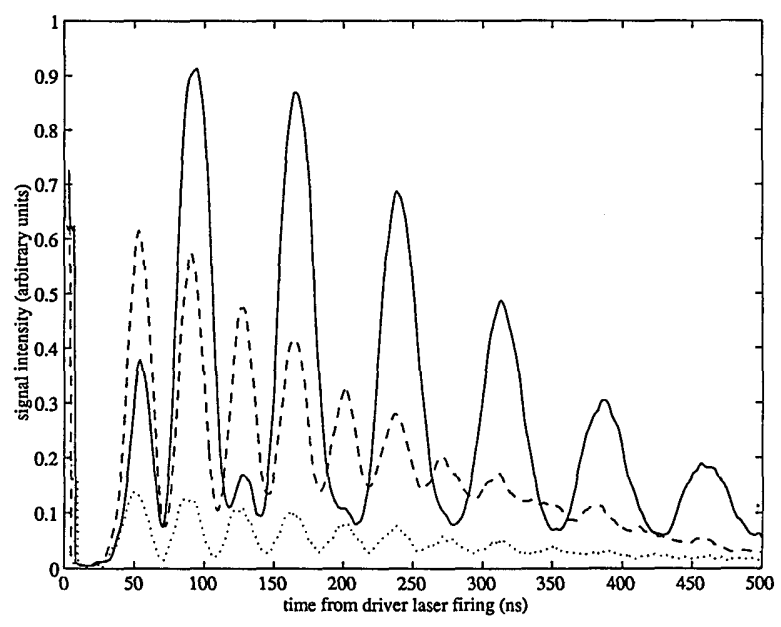

Fig. 2. Nonresonant (dotted curve), near-resonant (dashed curve), and resonant LITA (solid curve) signals. 


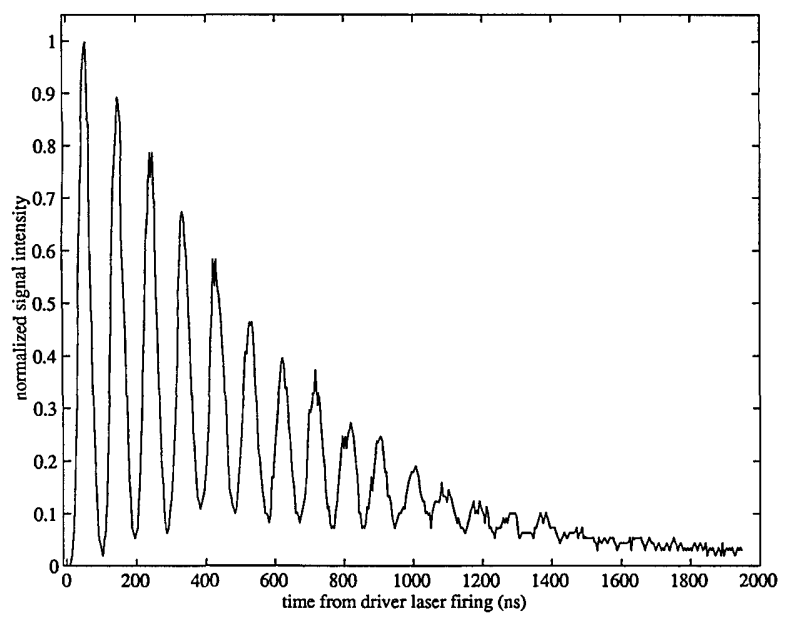

Fig. 3. Single-shot signal from $\mathrm{NO}_{2}$-seeded air obtained with a grating wavelength of $32.3 \mu \mathrm{m}$.

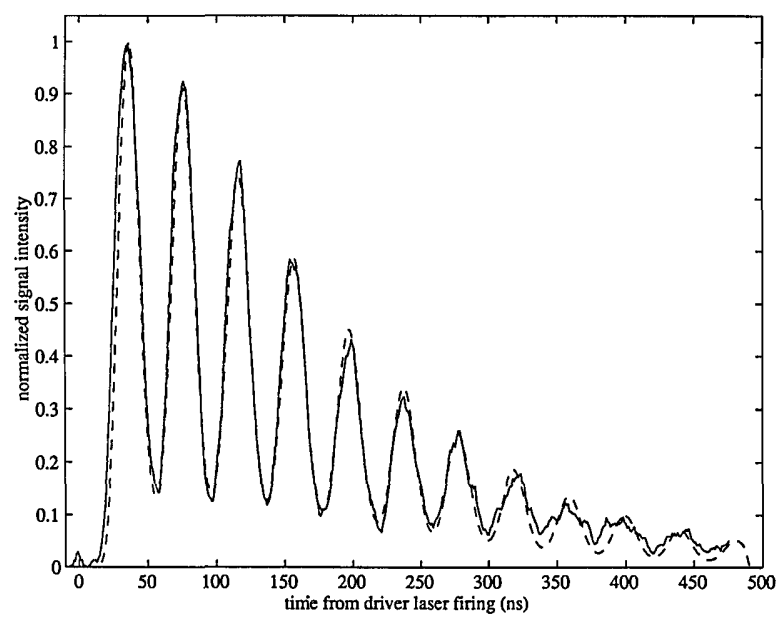

Fig. 4. Four-shot average signal from seeded air obtained with a grating wavelength of $14.5 \mu \mathrm{m}$ and theoretical fit (dashed curve).

enhanced signal recorded near a weak line of $\mathrm{NO}_{2}$ appears as a dashed curve in Fig. 2 for comparison. The signal recorded near the peak of the absorption line is the solid curve plotted in Fig. 3. On this curve the dominant modulation is at the Brillouin frequency, caused by interference between the signals scattered by the phonons and the thermon. LITA's good sensitivity is demonstrated since the ambient air contained less than 50 parts in $10^{9}$ of $\mathrm{NO}_{2}$ according to the Southern California Air Quality Management District.

Seeding the gas with $\mathrm{NO}_{2}$ vapors from a beaker of nitric acid provided LITA signals that were several orders of magnitude stronger than the signals obtained from room air, with estimated reflectivities near $10^{-4}$. A sample single-shot LITA signal appears in Fig. 3. The driver-beam crossing angle was $1.04^{\circ}$, yielding a grating wavelength of 32.3 $\mu \mathrm{m}$ and a Brillouin frequency of $10.7 \mathrm{MHz}$. At high concentrations of absorbing species, thermalization swamps electrostriction. Figure 4 shows a four-shot average of the LITA signal obtained when the beam-crossing angle was widened to $2.3^{\circ}$ (grating wavelength $14.5 \mu \mathrm{m}$; Brillouin frequency $23.9 \mathrm{MHz}$ ). Damping of the waves occurs on a time scale proportional to the square of the grating wavelength. In Fig. 4 the theoretical LITA signal is plotted with a dashed curve, showing excellent agreement with experiment. The sound speed and transport coefficients used in the fit are published values for dry air. Here, thermalization is resolved so the single-rate model of thermalization is replaced by the superposition of two-rate thermalizations: a fast $\sim 10-\mathrm{ns}$ process and a slow $\sim 100$-ns process. One could obtain a better fit to the data by adopting a more accurate thermalization model and source-beam profile model (a top-hat profile is assumed) and including the effect of source-beam phase noise.

In conclusion, LITA has demonstrated excellent sensitivity in the first round of experiments. In both magnitude and time history, the LITA signal is accurately represented by an analytical expression derived from the linearized equations of hydrodynamics and light scattering.

The author gratefully acknowledges the support of the University Research Initiative in aerothermochemistry sponsored by the U.S. Air Force Office of Scientific Research. The author is also indebted to the U.S. Office of Naval Research for its support of his graduate education through its fellowship program.

\section{References}

1. E. B. Cummings, GALCIT Rep. FM 92-2 (California Institute of Technology, Pasadena, Calif., 1992).

2. R. J. D. Miller, R. Casalegno, K. A. Nelson, and M. D. Fayer, Chem. Phys. 72, 371 (1982).

3. M. Buntine, D. Chandler, and C. Hayden, "Detection of excited-state absorption in water via laser-induced grating spectroscopy," J. Chem. Phys. (to be published).

4. R. W. Boyd, Nonlinear Optics (Academic, New York, 1992), Chap. 8, pp. 327-331.

5. B. J. Berne and R. Pecora, Dynamic Light Scattering (Wiley, New York, 1976), Chap. 10, pp. 229-232.

6. A. C. Eckbreth, Laser Diagnostics for Combustion Species and Temperature (Abacus, Cambridge, Mass., 1988), pp. 249-252. 